\title{
Rapport non traditionnel aux études : les règles des universités et de l'État québécois ${ }^{1}$
}

Mélanie Julien \& Lynda Gosselin Conseil supérieur de l'éducation

Résumé

Plusieurs étudiants entretiennent un rapport non traditionnelà leurs études en raison de l'occupation d'un emploi en parallèle, de responsabilités parentales, d'études à temps partiel ou d'un retour aux études après une interruption plus ou moins longue. Des actions sont mises en œuvre au sein des universités et de l'État québécois pour soutenir l'accès aux études et la réussite de ces étudiants : diversification de l'offre et des modalités de formation, ajout de dispositions à l'aide financière aux études, etc. À maints égards toutefois, les règles des universités et de l'État demeurent pensées pour les personnes dont le rapport aux études est traditionnel, de sorte qu'elles peuvent désavantager celles qui s'en éloignent.

\begin{abstract}
Many students today adopt a nontraditional relationship with their studies, namely by working while studying, balancing family responsibilities, enrolling part-time, or returning to them after a brief or long interruption. Some initiatives undertaken by Quebec universities and the province support access to and completion of studies for these students, notably the diversification of programs and learning formats, and new provisions for financial assistance. However, rules established by Quebec universities and the province are still based to a large extent on the traditional student model and, as such, can disadvantage people who move away from this model.
\end{abstract}

\section{Introduction}

Dans la foulée de la démocratisation de l'éducation amorcée au milieu du XX ${ }^{\mathrm{e}}$ siècle, la population étudiante des universités s'est pluralisée en ce qui a trait à ses caractéristiques sociodémographiques. Plus encore, cette population s'est transformée sur le plan des modalités de réalisation d'un projet d'études. Quatre réalités étudiantes témoignent éloquemment de cette transformation : le travail pendant les études, la parentalité des 
étudiants, les études à temps partiel et le retour aux études. Des données illustrent l’importance de ces réalités étudiantes à l'échelle du Québec. En 2006, dans le réseau de l'Université du Québec, $71 \%$ des nouveaux inscrits occupent un emploi et près du quart ont des responsabilités parentales (Bonin, 2007). Dans l'ensemble des universités québécoises à l'automne 2009, près du tiers des étudiants de premier cycle et de la moitié de ceux de deuxième cycle sont inscrits à temps partiel (CSE, 2013a). Au même trimestre, la proportion d'étudiants qui dépassent l'âge « limite » d'un parcours de formation régulier s'évalue à $20 \%$ au baccalauréat, à $33 \%$ à la maîtrise, et au-delà dans les programmes ne menant pas à un grade (CSE, 2013a).

Ces réalités étudiantes peuvent être appréhendées à partir de la notion de rapport aux études, laquelle est utilisée depuis le milieu des années 1990 en sociologie de l'éducation pour explorer l'hétérogénéité de la population étudiante. Comme le pose Dubet (1994) dans le contexte de la massification des universités françaises, " [c]'est dans les rapports des étudiants à leurs études eux-mêmes, plus que dans les facteurs «déterminants» (comme l'origine sociale), que l'on peut chercher les principes d'identification et de construction des expériences étudiantes » (p. 512). Selon les auteurs qui s’intéressent à la question, la notion de rapport aux études peut se rapporter au degré d'engagement dans les études (Dubet, 1994), à la nature du projet poursuivi (Dubet, 1994; Erlich, 2009), au rapport au savoir (Pralong, 1999) ou aux valeurs affichées à l'égard des études (Hamel, Méthot, \& Doré, 2011).

Dans le cadre des travaux rapportés ici, la notion de rapport aux études désigne « le rapport au métier, à la vie, à l'avenir, plus qu'un rapport au savoir justifiant le caractère instrumental du travail scolaire » (Erlich, 2009, p. 59). Cette acception tient compte de la part de rationalité de l'étudiant dans la construction de son parcours, en écho au courant de l'individualisme méthodologique en sciences sociales (Boudon, 1999). À cet égard, elle rejoint Pilote et Garneau (2011) pour qui le rapport aux études réfère au fait que « les jeunes exercent des choix et organisent hiérarchiquement les éléments de leur expérience étudiante en fonction de l'importance accordée » (p. 18). Plus précisément, le rapport aux études est conçu ici sous l'angle a) du mode d'engagement dans les études, selon la place qu'occupent ces dernières parmi les différentes sphères de la vie, et b) du mode de fréquentation de l'université, selon le degré de régularité du parcours de formation. Ainsi, un rapport aux études est dit traditionnel lorsque l'étudiant s’investit de façon quasi exclusive dans des études à temps plein, au cours d'un cheminement ininterrompu et linéaire. Par opposition, le rapport aux études est qualifié de « non traditionnel » en référence à un engagement non exclusif dans les études ou à un parcours de formation irrégulier. Un tel rapport aux études se manifeste notamment chez les étudiants qui occupent un emploi ou qui ont des responsabilités parentales (engagement non exclusif) et chez ceux qui étudient à temps partiel ou qui effectuent un retour aux études après une interruption plus ou moins longue (parcours irrégulier). Dans les faits, les réalités étudiantes qui fondent un rapport aux études non traditionnel s'entrecroisent, puisqu'elles peuvent caractériser une même personne au cours de son cheminement.

La notion de « rapport aux études non traditionnel » s'apparente à celle « d'étudiants non traditionnels » employée au Québec (Cloutier, 1990; Bonin, Bujold, \& Doray, 2012) comme ailleurs (Choy, 2002; Forbus, Newbold, \& Mehta, 2010; Kim, Sax, Lee, \& Hagedorn, 2010) pour désigner les étudiants qualifiés de « nouveaux » en raison de leurs caractéristiques sociodémographiques, de leur mode de vie ou de leur parcours de formation. Elle s'en 
distingue toutefois, car par-delà les caractéristiques de l'étudiant lui-même, elle est centrée sur son mode d'engagement dans les études et sur son parcours de formation. De fait, même des étudiants dont les caractéristiques sont traditionnelles adoptent un rapport aux études non traditionnel : c'est notamment le cas de jeunes qui concilient travail et études ou de ceux qui interrompent temporairement leur projet de formation (Cyr, 2006).

Diverses tendances sociales, économiques et culturelles peuvent expliquer l'essor du rapport aux études non traditionnel, à commencer par le rehaussement des exigences de scolarisation et la valorisation de la formation tout au long de la vie (UNESCO, 2005; Lévy, 1997). Par ailleurs, les pressions sociales relatives à l'autonomie individuelle, à la productivité et à la consommation incitent à occuper un emploi avant d'avoir achevé ses études (Roy, 2008), ce que permettent notamment les emplois non qualifiés à temps partiel dans le secteur de la vente et des services (Laplante, Street, Moulin, Doray, \& Verdy, 2010). L'adoption d'un rapport aux études non traditionnel fait aussi écho à la quête d'équilibre entre les sphères de la vie (Gauthier \& Vultur, 2007; Mercure \& Vultur, 2010). Elle est également liée à l'individualisation croissante des parcours de vie : l'étudiant a une marge de manœuvre pour façonner son parcours scolaire (Charbonneau, 2007), le travailleur devient l'entrepreneur de sa vie professionnelle (Bernier, 2011) et l'individu, l'auteur de sa propre vie (Beck, 2001). Bref, le rapport aux études non traditionnel s'enracine dans de profondes tendances. Il se présente comme un phénomène difficilement réversible, mais sur lequel des données et des analyses demeurent rares.

Pourtant, la prévalence du rapport aux études non traditionnel n'est pas sans ébranler le modèle d'une université conçue pour des jeunes engagés à temps plein de façon quasi exclusive dans un parcours de formation régulier. De la même manière, elle marque une rupture avec une vision séquentielle des étapes de la vie que sont les études, le travail et la parentalité. Il apparaît dès lors opportun d'examiner comment, dans le discours et la pratique, les universités et l'État québécois prennent en compte les réalités étudiantes qui fondent un rapport aux études non traditionnel. Soutiennent-ils l'accès aux études et la réussite des étudiants concernés de manière à assurer l'équité pour l'ensemble des personnes ayant la capacité et la volonté de poursuivre des études universitaires?

Pour répondre à cette question, le présent article se penche sur les règles qui, à l'échelle des universités et de l'État québécois, façonnent la composition de la population étudiante, ses conditions d'études ou ses conditions de vie. Entendue au sens sociologique, la notion de règles renvoie ici à l'ensemble des politiques, des règlements, des mesures, des programmes et des services qui guident la conduite des acteurs en établissant, de façon formelle ou non, des restrictions, des sanctions, des avantages, etc. À l'échelle des universités, elle réfère notamment aux règlements des études et aux services aux étudiants. À l'échelle de l'État, elle se rapporte aux politiques et aux programmes relatifs à l'enseignement universitaire (dont les mesures d'aide financière aux études), au soutien aux familles et à la parentalité, ainsi qu'au développement et à la formation de la maind'œuvre. C'est par l'analyse de ces règles que se révèlent des tendances quant à la manière dont les universités et l'État québécois prennent en compte le travail pendant les études, la parentalité des étudiants, les études à temps partiel et les retours aux études. Parce qu’il met en lumière certaines lacunes ou faiblesses, l'état des lieux qui en découle interpelle les administrateurs universitaires et les décideurs, de même que toute personne soucieuse de l'amélioration continue du système d'enseignement supérieur. 


\section{Méthode}

Menée à l'automne 2012 et à l'hiver 2013, l'analyse ${ }^{2}$ des règles des universités et de l'État québécois reposait sur cinq démarches complémentaires :

1. une recension des écrits;

2. un repérage et une analyse qualitative de documents publics (politiques, règlements, planifications stratégiques, rapports, etc.) relatifs aux 18 établissements universitaires québécois et au siège social del'Université du Québec : le corpus a été colligé par établissement à l'aide d'une grille de lecture permettant de structurer les renseignements se rapportant à l'offre de formation, aux modalités de formation, aux règles d'admission, de cheminement et de sanction, ainsi qu'aux conditions de vie des étudiants;

3. un repérage et une analyse qualitative de politiques et de programmes gouvernementaux québécois relatifs, entre autres, à l'enseignement et à la recherche ${ }^{3}$ universitaires, au soutien aux familles et à la parentalité, ainsi qu'au développement et à la formation de la main-d'œuvre;

4. une analyse quantitative de données extraites en 2011 du système de gestion de données de l'effectif étudiant (GDEU) du Ministère de l'Éducation, du Loisir et du Sport (MELS);

5. un relevé de documents produits par divers groupes et organismes.

Le portrait de situation dégagé du croisement de ces cinq démarches a fait l'objet d'une validation auprès d'experts et d'acteurs de l'enseignement supérieur, notamment auprès des membres de la Commission de l'enseignement et de la recherche universitaires, une instance du Conseil supérieur de l'éducation.

Cette analyse comporte des limites découlant principalement de la disponibilité des données et de leur nature variée. En particulier, le recours à des documents publics des universités, à des politiques et programmes gouvernementaux ainsi qu'à des rapports produits par des groupes d'intérêts pose des défis qui, comme le souligne Van der Maren (1996), relèvent de "la diversité des conditions ou des contextes dans lesquels ces documents ont été produits » (p. 302) et donc de la variété des intentions poursuivies par leurs auteurs. L'interprétation juste et cohérente des données colligées a exigé non seulement de les croiser, mais aussi de poser un regard critique sur chacune.

\section{Résultats}

\section{Regard sur les universités québécoises}

À travers leurs programmes d'études, leurs modalités de formation, leurs règles d'admission et de cheminement ainsi que leurs services et appuis aux étudiants, les universités influencent l'accès aux études et la réussite. Cette influence peut s'exercer de façon singulière dans le cas des étudiants dont le rapport aux études est non traditionnel.

L'offre de formation. Dans l'ensemble des universités québécoises, l'offre de formation a crû considérablement ces dernières années. Cette croissance se double d'une diversification des programmes d'études qui permet de rejoindre différentes populations, dont celles qui ont un rapport aux études non traditionnel. C'est le cas, par exemple, des maitrises et des doctorats axés sur des activités professionnelles, des programmes de per- 
fectionnement conçus pour des professionnels en exercice et des formations d'appoint destinées aux personnes ayant acquis une formation de niveau supérieur hors du Québec. Les universités offrent aussi un nombre croissant de programmes ne menant pas à un grade au premier et deuxième cycles (c'est-à-dire des microprogrammes comptant entre 9 et 15 crédits, ainsi que des certificats et diplômes comportant généralement 30 crédits), en particulier en administration et en éducation (voir le tableau 1). La grande majorité des étudiants qui s'y inscrivent cheminent à temps partiel (CSE, 2013a). Et bien qu'ils intéressent des étudiants de tous âges, ces programmes sont prisés par des individus plus âgés, qui effectuent possiblement un retour aux études (voir le tableau 2). Dans certains milieux universitaires, ces programmes s'accompagnent de pratiques de sanction particulières susceptibles de concerner des étudiants au parcours irrégulier : l'octroi d'un grade par cumul de diplômes (présent au $1^{\mathrm{er}}$ cycle et qui gagne le $2^{\mathrm{e}}$ cycle) et l'octroi d'une attestation, d'un certificat ou d'un diplôme pour des activités suivies dans un programme de grade non réalisé dans son entièreté.

Bon nombre d'étudiants dont le rapport aux études est non traditionnel se situent dans une perspective de formation continue. De nombreux documents des universités témoignent d'une volonté d'assurer le développement de ce type de formation tant sur le plan quantitatif (nombre de programmes et d'étudiants) que sur le plan qualitatif (qualité et pertinence des programmes); au moins deux universités se sont même dotées d'une politique en la matière.

Les modalités de formation. Dans les règlements des études et les brochures d'information, le régime d'études à temps partiel est souvent présenté comme une option, en particulier au premier cycle. Toutefois, l'inscription à temps plein demeure obligatoire, sinon encouragée, dans certaines disciplines (comme en médecine, en génie et en droit), de même que dans plusieurs formations à la recherche aux cycles supérieurs. Ainsi, la proportion d'étudiants à temps partiel varie selon les domaines et le type de programme (voir le tableau 3) : elle est généralement faible en sciences pures, en sciences appliquées et en sciences de la santé; dans les programmes de grade, elle est inférieure à celle observée dans les microprogrammes, certificats et diplômes. Par ailleurs, les universités réservent généralement aux étudiants à temps plein l'accès à plusieurs bourses institutionnelles; quelquesunes appliquent aussi cette restriction pour l'accès à des services de soutien psychologique ou d'aide individualisée. Néanmoins, les planifications stratégiques de quelques universités ciblent le recrutement, l'accès aux services ou la persévérance des étudiants à temps partiel.

Le recours à des plages horaires variées, à la formation à distance et à la formation en dehors des campus principaux sont autant de modalités de formation qui peuvent soutenir l'accès aux études et la réussite d'étudiants qui entretiennent un rapport aux études non traditionnel. Dans l'ensemble, les universités recourent de plus en plus aux cours du soir, aux sessions intensives (un week-end par mois ou deux semaines consécutives, par exemple, surtout dans le cadre de programmes ne menant pas à un grade) et au trimestre d'été. Malgré des différences significatives entre les universités, l'offre de cours offerts à distance est en croissance et figure dans les perspectives de développement de plusieurs établissements. Pour ce qui est de la délocalisation, plus de 200 lieux d'enseignement sont dénombrés pour les 18 universités québécoises (CREPUQ, 2010; Crespo, BeaupréLavallée, \& Dubé, 2011). 


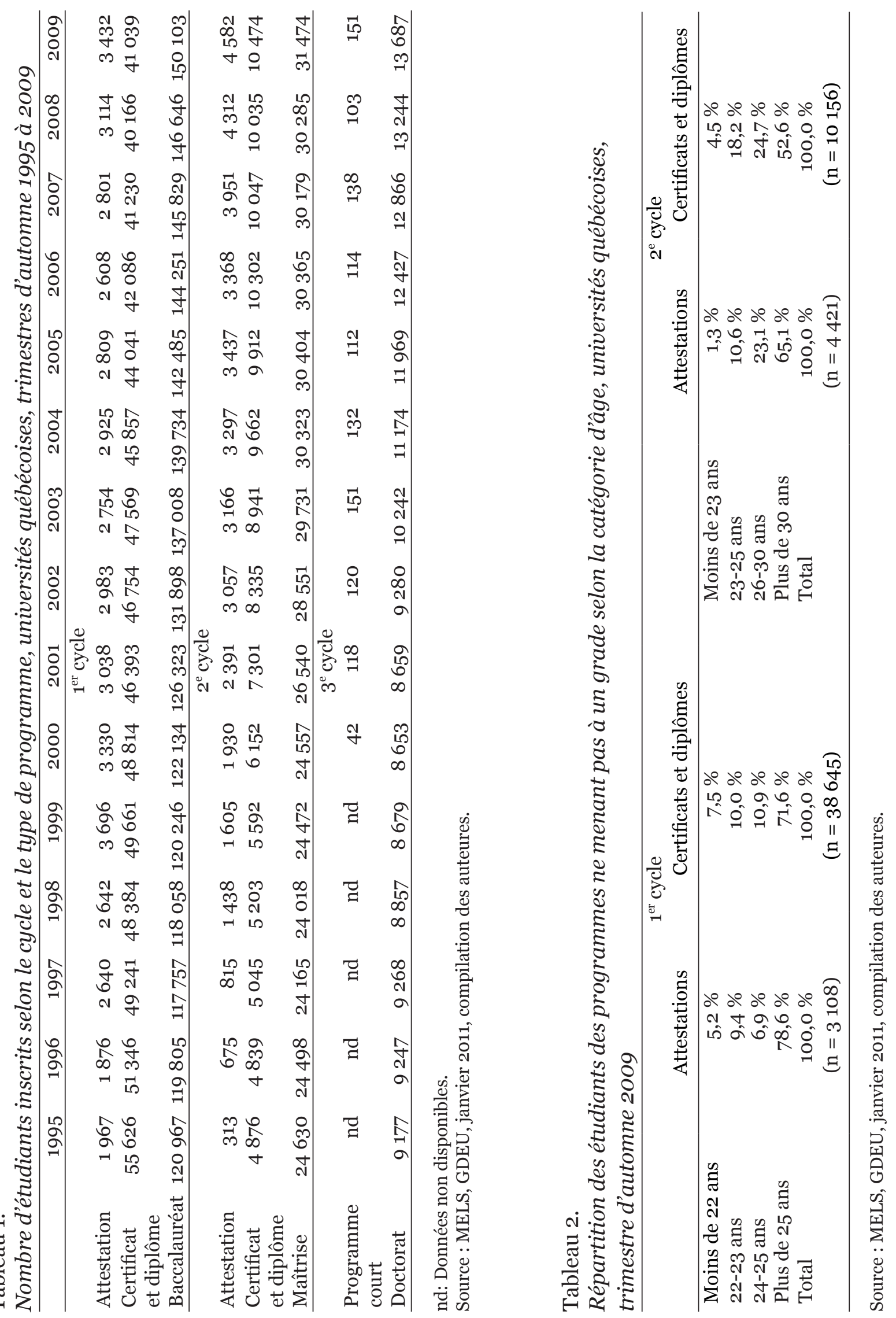




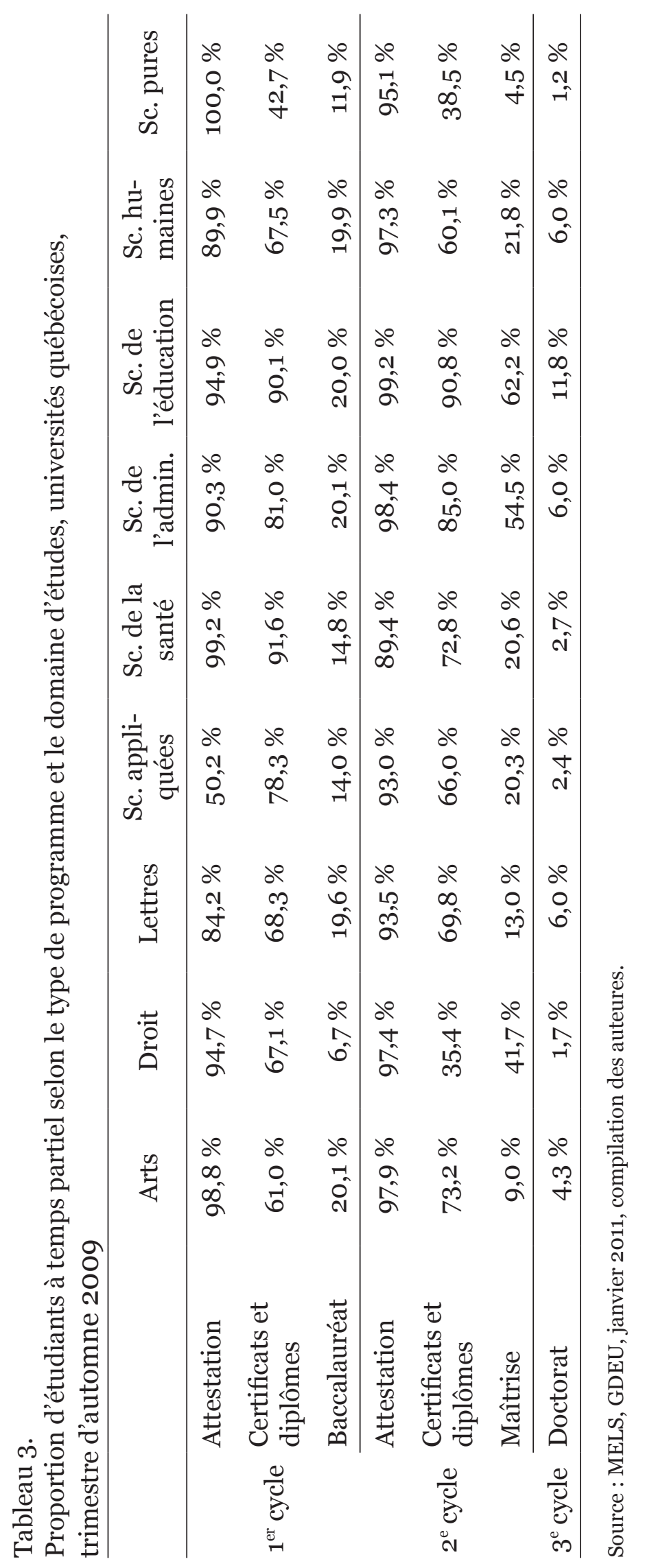


Des règles d'admission et de cheminement. Les universités peuvent admettre des candidats non titulaires d'un diplôme du niveau directement inférieur (ou l'équivalent) à celui recherché, ce qui permet d'accueillir des personnes qui souhaitent effectuer un retour aux études. Les critères d'une telle admission (nommée couramment " admission sur une base adulte ») reposent généralement sur l'âge (avoir 21 ou 23 ans), la durée de l'interruption des études (avoir cessé d'étudier pendant un, deux, voire cinq ans) et l'expérience (avoir des aptitudes particulières ou de l'expérience dans un domaine). L'Association canadienne d'éducation des adultes des universités de langue française (ACDEAULF, 2006) évaluait à $20 \%$ la proportion d'étudiants des universités québécoises qui, en 2002-2003, étaient admis selon ces critères, soit $22 \%$ au premier cycle et $13 \%$ au deuxième cycle. Selon le Groupe de travail sur la réalité de l'adulte à l'Université Laval (2007), des programmes « n'en acceptent que rarement : architecture, ergothérapie, kinésiologie, nutrition et physiothérapie », alors que d'autres " les refusent : médecine, médecine dentaire et pharmacie » (p. 26). Une reconnaissance des acquis (scolaires ou extrascolaires) et des compétences (RAC) permet l'octroi d'équivalences pour des cours dont les objectifs ont déjà été atteints, disposition qui touche particulièrement les étudiants dont le parcours est irrégulier. Les règlements des études limitent la proportion de crédits pouvant faire l'objet d'une telle reconnaissance : le plus souvent aux deux tiers des crédits d'un programme de grade et à la moitié des crédits d'un certificat ou d'un diplôme. L'analyse montre que la RAC aux fins de reconnaissance de crédits demeure toutefois exceptionnelle comparativement à celle réalisée aux fins d'admission, bien que des volontés d'y avoir davantage recours s'expriment dans plusieurs universités (GRAC, 2010) et même à l'échelle du gouvernement (MESRST, 2013).

Des dispositions prévoyant l'autorisation de la direction de programme ou de recherche permettent à l'étudiant d'interrompre temporairement sa formation en raison, notamment, d'obligations professionnelles et parentales. Des « congés parentaux » sont prévus dans quelques règlements des études, pour un ou deux trimestres ou au plus 24 mois. Quant aux absences ponctuelles à un cours dues à des obligations parentales, elles sont rarement balisées : elles sont presque toujours soumises à la seule appréciation du professeur.

Les services et appuis aux étudiants. Les universités québécoises mènent des activités spécifiques pour le recrutement, l'accueil et le soutien des personnes qui envisagent un retour aux études. En plus des séances d'information et des messages publicitaires, certaines universités disposent d'unités dédiées à la formation continue pour fournir aux candidats un soutien particulier.

Pour ce qui est de la parentalité des étudiants, des associations étudiantes pour les étudiants-parents ont récemment vu le jour dans certaines universités. Des services de garde existent sur quelques campus, mais ils sont généralement réservés aux enfants des membres de toute la communauté universitaire (employés et étudiants). Des services de halte-garderie, mieux adaptés aux horaires atypiques, n'existent que dans un seul établissement, bien que le projet d'en mettre en place soit exploré dans au moins trois lieux.

Quant au travail pendant les études, l'analyse montre qu'il est valorisé au sein des universités. En témoignent principalement : 1) les stages rémunérés prévus dans plusieurs programmes d'études, 2) le fait que les services de placement s'adressent non seulement aux nouveaux diplômés, mais aussi aux étudiants, 3) l'embauche d'étudiants pour des 
tâches de soutien à l'enseignement et à la recherche, et 4) le Programme études-travail de l'Aide financière aux études ${ }^{4}$. En outre, onze syndicats regroupant des étudiants employés par une université québécoise ont été créés entre 2005 et 2013. Bien qu'il soit reconnu, le travail étudiant fait l'objet d'actions visant à le baliser. Par exemple, le premier article des conventions collectives des syndicats des étudiants porte souvent sur la priorité à accorder aux études.

Vue d'ensemble. Le degré de prise en compte des réalités étudiantes qui fondent un rapport aux études non traditionnel varie d'une université à l'autre, voire d'une unité d'enseignement à l'autre, ce qui peut s'expliquer par les spécificités de la mission et de la population étudiante de chacune. En effet, bon nombre d'universités ont une mission particulière dans laquelle s'enracine une préoccupation historique pour ces réalités, alors que quelques-unes manifestent ces dernières années une volonté d'actualiser leurs pratiques en fonction de nouveaux profils et parcours des étudiants. En revanche, une minorité paraît particulièrement attachée aux modes traditionnels de fréquentation de l'université et d'engagement dans les études.

C'est principalement par l'offre et les modalités de formation que les universités soutiennent l'accès aux études et la réussite des étudiants qui entretiennent un rapport aux études non traditionnel. De façon moins marquée, les règles d'admission et de cheminement, de même que les services aux étudiants, y participent aussi; des souhaits d'amélioration ont néanmoins été repérés, notamment en matière de reconnaissance des acquis et des compétences, ainsi que de services de garde.

Enfin, l'analyse révèle que, dans l'ensemble, les universités consentent des efforts plus soutenus pour favoriser et encadrer le travail pendant les études que pour prendre en compte les conditions particulières des étudiants-parents. Ce constat peut s'expliquer par le fait que l'occupation d'un emploi est plus fréquente que les responsabilités parentales, qu'elle peut représenter un moyen d'acquérir des connaissances et des compétences associées à la formation, et que l'embauche d'étudiants fait partie de l'histoire du système universitaire québécois.

\section{Regard sur l'État québécois}

Les politiques et programmes gouvernementaux influencent la composition de la population étudiante, de même que ses conditions d'études et de vie. C'est notamment le cas de ceux qui se rapportent à l'enseignement et à la recherche universitaires, au soutien aux familles et à la parentalité, ainsi qu'à la formation et au perfectionnement de la maind'œuvre.

Les universités et leur financement. Dans la Politique québécoise à l'égard des universités (MEQ, 2000a), l'État promeut un accès large et démocratique à l'université, tout en insistant sur les notions d'aptitudes, de volonté et d'engagement des étudiants. Certaines réalités étudiantes qui fondent un rapport aux études non traditionnel font partie des préoccupations qui y sont évoquées, mais elles y occupent une place restreinte. De surcroît, elles y font l'objet d'une représentation tronquée. Par exemple, les études à temps partiel sont associées seulement aux étudiants plus âgés et à ceux inscrits dans les programmes ne menant pas à un grade, ces derniers étant essentiellement considérés comme des voies de perfectionnement des diplômés. Ce constat fait écho à l'ACDEAULF (2001), qui déplore que la Politique soit « restée désespérément muette » (p.4) en matière 
de formation continue, et à Doray et Mayrand (2001) pour qui « globalement, la question de la formation des adultes à l'université [y] est peu couverte » (p. 12).

Prévue dans la Politique québécoise de financement des universités (MEQ, 200ob), la formule de financement des universités se base principalement sur l'effectif étudiant en équivalence au temps plein (EEETP) et sur le coût estimé de la formation. Elle comporte ainsi des défis particuliers pour les établissements qui accueillent une proportion élevée d'étudiants à temps partiel (Commission de la culture et de l'éducation, 2011). Par ailleurs, le financement des microprogrammes, des certificats et des diplômes est proportionnellement équivalent à celui des programmes menant à un grade, mais un financement moindre ou conditionnel à la diplomation des étudiants inscrits à de tels programmes a déjà été envisagé par le gouvernement. Même si cette avenue a été déconseillée par le Groupe de travail sur le financement des universités (1997), elle demeure présente dans le paysage de l'enseignement supérieur québécois, comme en font foi les rapports de Bissonnette et Porter (2013), et de Tremblay et Roy (2013).

Les droits de scolarité et l'aide financière aux études. Si les droits de scolarité sont les mêmes pour tous les étudiants qui résident au Québec, des groupes ont déjà proposé leur modulation selon que l'étudiant emprunte ou non un parcours de formation régulier (Groupe de travail sur le financement des universités, 1997; FCCQ, 2013). Bien qu'une telle modulation suscite une vive opposition (FAEUQEP, 2004), son évocation rappelle que, pour l'heure, la valeur des parcours de formation irréguliers n'est pas reconnue par tous.

En ce qui concerne l'Aide financière aux études (AFE), la très grande majorité des étudiants à temps partiel n'auraient pas accès au Programme de prêt pour les études à temps partiel (PPETP) instauré en 2002, puisque n'y sont pas admises les personnes ayant des revenus annuels supérieurs à 13000 \$ et celles inscrites à un seul cours par trimestre (MQAF et FAEUQEP, 2011). De plus, non seulement l'aide fournie est modeste, soit un prêt de 1120 \$ en moyenne par année (CCAFE, 2009), mais le PPETP est indépendant du Programme de prêts et bourses (PPB), ce qui pose certaines difficultés aux personnes qui changent de régime d'études au cours de leur programme (Comité sur l'accessibilité financière aux études de l'Université du Québec, 2011). De surcroît, les étudiants qui passent du temps plein au temps partiel doivent prendre leur prêt en charge (CCAFE, 2009) et ne sont pas admissibles au Programme de remise de dette ${ }^{5}$, ni au Programme études-travail (abordé précédemment).

Quant aux bénéficiaires de l'AFE qui interrompent temporairement leurs études en raison d'une grossesse ou de la naissance ou de l'adoption d'un enfant, ils peuvent bénéficier d'un report du remboursement de leur dette d'études. Même inscrits aux études à temps partiel, les femmes enceintes, les parents avec conjoint dont l'enfant a moins de 6 ans et les chefs de famille monoparentale dont l'enfant a moins de 12 ans peuvent avoir le statut d'étudiant à temps plein aux fins d'admission à l'AFE. Malgré ces dispositions, les besoins des étudiants-parents ne sont pas entièrement pris en compte par l'AFE, notamment parce que certaines dépenses admises ne correspondent pas aux coûts réellement assumés (CCAFE, 2004; CSF, 2004; FAFMRQ, 2013; AELIES, 2013).

Du fait qu'elle prend en compte le salaire gagné au cours des huit mois précédant l'inscription aux études, l'AFE paraît moins généreuse queles programmes correspondants ailleurs au Canada en ce qui concerne les personnes qui effectuent un retour aux études 
(CCAFE, 2004). Celles-ci peuvent certes recourir au Régimed'encouragement àl'éducation permanente (REEP), qui permet de puiser dans un Régime enregistré d'épargne-retraite (REER) pour financer un retour aux études, mais cette mesure fiscale demeure très peu connue (CCAFE, 2009; MQAF et FAEUQEP, 2009).

Le financement de la recherche. Pour obtenir une bourse d'excellence des organismes subventionnaires ${ }^{6}$, la qualité du dossier académique et du projet de recherche de l'étudiant semble primer, selon les critères énoncés dans les différents programmes. Sont néanmoins valorisées les expériences de travail, dans la mesure où elles se situent dans le domaine de la recherche. L'expérience doit idéalement être antérieure à la maîtrise, ce que rendent possible les stages d'initiation à la recherche au premier cycle offerts par certains organismes subventionnaires. Conditionnellement à l'approbation de leur directeur ou de leur université, les boursiers peuvent suspendre le versement de leur bourse pendant une session pour acquérir une expérience pertinente de travail. Dans certains domaines, des bourses de recherche en milieu de pratique sont offertes dans le cadre de partenariats entre une université et une entreprise ou un organisme. En outre, les programmes de bourses légitiment la pratique qui veut qu'un étudiant « travaille » pour son directeur; ils établissent toutefois un nombre d'heures de travail maximal pour que la priorité soit accordée aux études.

Les boursiers doivent généralement être inscrits aux études à temps plein. Certains organismes prévoient expressément qu'un étudiant perde sa bourse s'il décide de poursuivre ses études à temps partiel. Des possibilités d'études à temps partiel en raison d'obligations parentales ont toutefois été repérées en sciences de la nature et des technologies, sous réserve de l'autorisation de l'université, du centre de recherche ou du directeur de recherche.

Par ailleurs, les boursiers qui interrompent leurs études en raison de la naissance ou de l'adoption d'un enfant peuvent bénéficier d'un « congé parental sans solde » (généralement d'une durée maximale de 12 mois pendant lesquels le versement de la bourse est reporté) ainsi que d'un « congé parental payé » (de 4 ou 6 mois). Cette dernière disposition « ne peut [toutefois] être vue comme une mesure de soutien financier au rôle parental en bonne et due forme puisqu'elle ne consiste pas à ajuster le montant des bourses en fonction des charges financières que représente un enfant »(CSF, 2004, p. 21). Dans tous les cas, l'autorisation du directeur est requise.

Dans l'ensemble, les programmes de bourses tendent à privilégier les étudiants dont le parcours de formation est linéaire et sans interruption, hormis dans le cas des bourses de réintégration offertes par le FRQNT, le FRQSC et le FRQS en vue d'encourager les retours aux études des personnes qui ont obtenu un diplôme de $1^{\mathrm{er}}$ ou de $2^{\mathrm{e}}$ cycle depuis quelques années et qui désirent poursuivre des études de maîtrise ou de doctorat.

La parentalité et la famille. Le Régime québécois d'assurance parentale (RQAP) s'adresse aux salariés, à temps plein ou à temps partiel. N'y sont donc pas admissibles les non-salariés, dont les étudiants qui n’ont pas travaillé pendant l'année précédant la naissance ou l'adoption d'un enfant, ni les étudiants et les stagiaires postdoctoraux qui ont bénéficié d'une bourse ou d'un revenu issu d'une subvention de recherche d'un professeur. Cette situation fait l'objet de maintes critiques (AELIES, 2013; FAFMRQ, 2013; CNCS-FEUQ, 2010; CCJ et CNCSFEUQ, 2010; CFE, 2008; CSF, 2004). 
Par ailleurs, comme plusieurs parents québécois, les étudiants-parents peinent à obtenir des places dans les services de garde subventionnés. Certains éprouveraient des difficultés supplémentaires, en raison de leur horaire qui correspond plus difficilement à celui des services de garde conventionnels (Conseil d'intervention pour l'accès des femmes au travail, 2006). Plusieurs demandes relatives à l'augmentation du nombre de places en service de garde et à la création de haltes-garderies sont d'ailleurs formulées par différents groupes (CCJ et CNCS-FEUQ, 2010; CSF, 2004).

Enfin, la Politique et les Plans d'action sur l'égalité entre les hommes et les femmes (Gouvernement du Québec, 2007a, 2007b, 2011) prévoient nettement plus d'actions pour la conciliation travail-famille que pour la conciliation études-famille. Les quelques passages qui portent sur cette dernière en milieu universitaire sont essentiellement destinés à informer les étudiants-parents des mesures en place et à mieux connaître leurs besoins. Des groupes ont d'ailleurs déploré le peu d'engagement de l'État à l'égard de la conciliation études-famille (CSF, 2004; CCJ et CNCS-FEUQ, 2010).

La formation en emploi et le développement de la main-d'œuvre. Les mesures dédiées à la formation de la main-d'œuvre concernent une formation universitaire dans moins de 0,5 \% des cas (MESS, 2012). De plus, celle-ci n'est que très rarement ciblée par des projets financés dans le cadre de la Loi favorisant le développement et la reconnaissance des compétences de la main-d'œuvre (communément appelée la Loi du $1 \%$ ). Quant à la Politique gouvernementale d'éducation des adultes et de formation continue (MEQ, 2002b), elle concerne expressément la formation de base, sans référence au rôle des universités en matière de formation continue. Dans le plan d'action qui l'accompagne (MEQ, 2002a), des responsabilités leur sont tout de même dévolues en matière de formation d'appoint de personnes immigrantes et de reconnaissance des acquis et des compétences. Cette modeste attention pour la formation continue à l'enseignement universitaire est critiquée, notamment par des ordres professionnels pour qui « l'implication des établissements d'enseignement, dont les universités, est [...] essentielle » (CIQ, 2004, p. 8-9) pour assurer la formation continue des professionnels en exercice.

Dans un autre ordre d'idées, l'État favorise l'emploi pendant les études au moyen du site Web Placement étudiant d'Emploi-Québec (qui répertorie les emplois disponibles dans les secteurs privé et public) et de différents programmes qui permettent l'embauche d'étudiants et de stagiaires au sein des ministères et organismes, en particulier pendant la période estivale. En parallèle, promues dans le Pacte pour l'emploi (MESS et MELS, 2008), des initiatives naissent, notamment en Estrie et au Saguenay-Lac-Saint-Jean, en vue de sensibiliser les employeurs aux exigences que pose la réussite d'un projet d'études.

Vue d'ensemble. Dans les règles étatiques, la façon traditionnelle de réaliser un projet d'études universitaires semble prédominer, hormis pour ce qui est de l'occupation d'un emploi. De fait, les parcours de formation enrichis par des expériences professionnelles sont valorisés.

Certaines règles sont prévues pour les étudiants dont le rapport aux études est non traditionnel, mais elles se trouvent en marge des mesures existantes plutôt qu'intégrées dans une conception actualisée des populations étudiantes. De plus, des situations intermédiaires semblent échapper à l'action de l'État. Par exemple, les étudiants-parents peuvent glisser entre le champ de l'éducation et celui de la famille. Ce sont donc souvent aux professeurs qu'incombe la responsabilité de décider de la façon de composer avec certaines situations qui découlent de la parentalité des étudiants. 


\section{Discussion et conclusion}

En ce qui a trait au travail pendant les études, les universités et l'État véhiculent l'idée qu'il est souhaitable, en particulier s'il est lié au domaine d'études. L'acquisition d'expériences de travail est institutionnalisée au sein des universités, notamment dans les charges de cours et les tâches d'assistanat qui sont d'ailleurs inhérentes aux activités universitaires. Pour l'État, les étudiants représentent une main-d'œuvre essentielle à l'économie québécoise, ce qui se reflète notamment dans l'existence du site Web Placement étudiant. L'équilibre entre les études et le travail constitue néanmoins une source de préoccupation : des balises relatives au nombre maximum d'heures de travail par semaine sont énoncées et des activités de sensibilisation sont menées auprès d'employeurs.

Pour ce qui est de la parentalité, les mesures prévues par l'État suggèrent qu'il vaut mieux avoir un enfant une fois la carrière amorcée : dédié aux salariés, le RQAP est pensé en fonction de la séquence " études-travail-famille ». Quant aux récentes dispositions mises en place par les universités et l'État pour les étudiants-parents, elles s'ajoutent à la pièce sans véritable articulation entre elles. C'est à l'étudiant de les agencer au mieux, voire de planifier les naissances en conséquence, autant que faire se peut. Si la conciliation travail-famille fait de plus en plus l'objet de préoccupations sociales, il en va autrement de la conciliation études-famille qui, quoique depuis peu et de façon encore modeste, semble néanmoins interpeller les universités qui y sont confrontées au quotidien.

Les étudiants à temps partiel sont parfois considérés comme des étudiants « de second ordre ». L'inscription à certains programmes et l'accès à des bourses institutionnelles, voire l'accès à quelques services, sont réservés aux étudiants à temps plein. Les règles de financement des universités actuellement en vigueur ne favorisent pas une plus grande ouverture aux inscriptions à temps partiel. En outre, le fait d'étudier à temps partiel rend difficile l'accès à du soutien financier étatique, tant de l'AFE que des organismes subventionnaires de la recherche.

En ce qui concerne les retours aux études, les règles étatiques portent surtout sur la formation de base, dans la perspective d'assurer l'employabilité de personnes peu qualifiées. Le rôle confié aux universités en matière de formation continue reste ténu. De leur côté, les universités favorisent les retours aux études, notamment au moyen de l'offre et des modalités de formation. Les programmes ne menant pas à un grade y contribuent particulièrement, mais leur valeur est parfois remise en question.

Par-delà ces constats relatifs à chacune des réalités étudiantes qui fondent un rapport aux études non traditionnel, force est de souligner la quantité de règles fixées par les universités ou l'État québécois qui sont susceptibles d'influencer les modes de fréquentation de l'université et d'engagement dans les études. Comme on a pu le constater dans la démarche de validation, certaines de ces règles restent méconnues des professeurs et, a fortiori, des étudiants. Qui plus est, des divergences s'observent entre les orientations que promeuvent différentes règles : par exemple, l'AFE offre un statut particulier aux étudiants à temps partiel qui ont des responsabilités parentales, alors que certains programmes d'études ne permettent pas l'inscription à temps partiel. Sur quelques sujets comme les absences ou les interruptions d'études pour des raisons parentales, les règles laissent souvent les décisions au seul jugement des professeurs. C'est dire que le système universitaire québécois n'est pas toujours au clair quant à la façon de prendre en compte les réalités étudiantes qui fondent un rapport aux études non traditionnel. 
Somme toute, les règles qui soutiennent l'accès aux études et la réussite des personnes concernées par un rapport aux études non traditionnel demeurent, à maints égards, insuffisantes. Malgré des initiatives, les universités et, davantage encore, l'État québécois restent attachés à la vision d'un rapport aux études traditionnel, qui concerne pourtant une proportion décroissante de la population étudiante. Ce hiatus n'est pas sans conséquence : parce qu'en général les règles sont pensées pour les personnes dont le rapport aux études est traditionnel, elles sont susceptibles de désavantager celles qui s'en éloignent. Des efforts restent donc à faire pour soutenir l'accès aux études et la réussite de toutes les personnes qui ont la capacité et la volonté de réaliser un projet de formation universitaire. De tels efforts devront s'appuyer sur des activités de veille et de recherche permettant de mieux connaître les populations étudiantes, les pratiques qui ont cours au sein des universités, de même que l'effet des politiques et des programmes gouvernementaux sur l'accès aux études, les conditions d'études et les conditions de vie des étudiants.

\section{Notes}

1. Les auteures tiennent à remercier les deux évaluateurs qui, grâce à leurs judicieux commentaires, ont contribué significativement à l'amélioration de cet article.

2. Réalisée dans le cadre de la production d'un avis du Conseil supérieur de l'éducation (CSE, 2013a), cette analyse a donné lieu à un rapport de recherche (CSE, 2013b) dans lequel sont détaillés la méthodologie, les sources de données et les résultats.

3. Les programmes de recherche des organismes subventionnaires canadiens ont aussi été pris en compte.

4. Créé en 1999, ce programme permet à des étudiants qui éprouvent des difficultés financières d'obtenir un emploi dans leur établissement. En 2008-2009, près de 4900 étudiants de l'enseignement collégial ou universitaire en ont bénéficié (FEUQ, 2011). L'évaluation de ce programme montre qu'il est apprécié, mais insuffisant (MELS, 2007).

5. Le Programme de remise de dette permet de réduire de $15 \%$ la dette de toute personne qui a terminé ses études dans les délais prévus et qui a reçu une bourse chaque année dans le cadre du Programme de prêts et bourses (PPB).

6. FRQS, FRQNT et FRQSC à l'échelle du Québec, et IRSC, CRSNG et CRSH à l'échelle canadienne.

\section{Références}

Association canadienne d'éducation des adultes des universités de langue française (ACDEAULF). (2001). Notes à l'intention du Conseil supérieur de l'éducation en vue de la préparation de son rapport annuel portant sur le rôle de l'État et l'orientation de son intervention en éducation. Québec, Québec.

Association canadienne d'éducation des adultes des universités de langue française (ACDEAULF). (2006). La reconnaissance des acquis dans les universités québécoises : état de la situation. Québec, Québec.

Association des étudiantes et des étudiants de Laval inscrits aux études supérieures (AELIES).(2013).Dansl'optique d'uneréforme:visionétudiante del'institutionuniversitaire québécoise. Mémoire déposé au Sommet sur l'enseignement supérieur, Québec, Québec. 
Beck, U. (2001). La société du risque. Sur la voie d'une autre modernité. Paris, France : Aubier.

Bernier, C. (2011). Formation et employabilité : regard critique sur l'évolution des politiques de formation de la main-d'œuvre au Québec. Québec, Québec : Presses de l'Université Laval.

Bissonnette, L., \& Porter, J. R. (2013). L’Université québécoise : Préserver les fondements, engager des refondations. Rapport du chantier sur une loi-cadre des universités, Québec, Québec.

Bonin, S. (2007). Le projet ICOPE : prise de vue récente sur la conciliation étudestravail-famille. Repéré à : http://www.uquebec.ca/capres/articles/articles-07-08/vsimprimables/art-UQ-Novembre-2007.pdf.

Bonin, S., Bujold J., \& Doray, P. (2012). Universités nouvelles, étudiants nouveaux? Dans L. Roy \& Y. Gingras (dir.), Les universités nouvelles. Enjeux et perspectives (p. 277304). Québec, Québec : Presses de l'Université Laval.

Boudon, R. (1999). Individualisme méthodologique. Dans A. Akoun \& P. Ansart (dir.), Dictionnaire de sociologie (p. 277-279). Paris, France : Le Robert /Seuil.

Charbonneau, J. (2007). L'influence du contexte sociétal sur les trajectoires scolaires et professionnelles des jeunes adultes. Dans S. Bourdon \& M. Vultur (dir.), Regard sur... les jeunes et le travail (p. 53-68). Québec, Canada : Éditions de l'IQRC.

Choy, S. (2002). Nontraditional Undergraduates (NCSE 2002-012). Washington, DC : Department of education, National Center for Education Statistics.

Cloutier, R. (1990). Les « nouvelles » clientèles universitaires québécoises : différences et ressemblances avec le modèle de «l'étudiant traditionnel ». Québec, Québec : Université Laval.

Comité consultatif Jeunes, et Conseil national des cycles supérieurs - Fédération étudiante universitaire du Québec (CCJ, et CNCS-FEUQ). (2010). La conciliation familiale chez les jeunes. Montréal, Québec.

Comité consultatif sur l'accessibilité financière aux études (CCAFE). (2004). L'accessibilité financière à la réussite du projet d'études. Sainte-Foy, Québec.

Comité consultatif sur l'accessibilité financière aux études (CCAFE). (2009). Vers l'accessibilité financière à l'apprentissage tout au long de la vie. Québec, Québec.

Comité sur l'accessibilité financière aux études de l'Université du Québec. (2011). La participation aux études universitaires dans un contexte de hausse des droits de scolarité. Québec, Québec.

Commission de la culture et de l'éducation. (2011). Rapport sur les auditions des dirigeants des établissements d'enseignement de niveau universitaire en application de la Loi sur les établissements de niveau universitaire. Québec, Québec.

Conférence des recteurs et des principaux des universités du Québec (CREPUQ). (2010). L'offre de formation universitaire sur le territoire québécois : état de situation au trimestre d'automne 2008. Montréal, Québec. 
Conseil de la famille et de l'enfance (CFE). (2008). La politique familiale au Québec : visée, portée, durée et rayonnement. Québec, Québec.

Conseil d'intervention pour l'accès des femmes au travail. (2006). Personne ne doit choisir entre la famille et le travail! Un regard féministe sur la conciliation familleemploi-études. Montréal, Québec.

Conseil du statut de la femme (CSF). (2004). Étudiante et mère : un double défi - Les conditions de vie et les besoins des mères étudiantes. Québec, Québec.

Conseil interprofessionnel du Québec (CIQ). (2004). Mémoire à la Commission parlementaire de l'éducation dans le cadre de la consultation générale sur les enjeux entourant la qualité, l'accessibilité et le financement des universités. Montréal, Québec.

Conseil national des cycles supérieurs - Fédération étudiante universitaire du Québec (CNCS-FEUQ). (2010). Avis sur l'élargissement du régime d'assurance parentale aux étudiants de cycles supérieurs : passer du discours à l'action. Montréal, Québec.

Conseil supérieur de l'éducation (CSE). (2013a). Parce que les façons de réaliser un projet d'études universitaires ont changé... Québec, Québec.

Conseil supérieur de l'éducation (CSE). (2013b). Comment l'État et les établissements abordent-ils les réalités étudiantes actuelles? Document complémentaire à l'avis du Conseil. Québec, Québec.

Crespo, M., Beaupré-Lavallée, A., \& Dubé, S. (2011). L'offre de programmes universitaires des sites hors campus au Québec : ampleur, logiques décisionnelles et évaluation de ses impacts. Montréal, Québec : CIRANO.

Cyr, C. (2006). Une analyse descriptive de la progression des populations étudiantes de premier cycle à l'université (Mémoire de maîtrise). Université du Québec à Montréal. Repéré à : http://www.archipel.uqam.ca/3168/1/M9454.pdf.

Doray, P., \& Mayrand, P. (2001, février). Une innovation institutionnelle à l'université : la participation des adultes. Communication présentée au $35^{\mathrm{e}}$ colloque annuel de l'ACDEAULF, Ottawa, Ontario.

Dubet, F. (1994). Dimensions et figures de l'expérience étudiante dans l'université de masse. Revue française de sociologie, XXXV, 511-532.

Erlich, V. (2009). Un renouvellement du monde étudiant. Dans B. Roudet (dir.), Les jeunes en France (p. 43-68). Québec, Québec : Presses de l'Université Laval.

Fédération des associations de familles monoparentales et recomposées du Québec (FAFMRQ). (2013). Études et monoparentalité : trop de devoirs! Document préparé dans le cadre du Sommet sur l'enseignement supérieur, Montréal, Québec.

Fédération des associations étudiantes universitaires québécoises en éducation permanente (FAEUQEP). (2004). Une université pour tous : un droit. Mémoire présenté à la Commission parlementaire sur la qualité, l'accessibilité et le financement des universités, Montréal, Québec.

Fédération des chambres de commerce du Québec (FCCQ). (2013). L'enseignement supérieur: accessibilité, qualité, diversité. Mémoire présentéau Sommet surl'enseignement supérieur, Montréal, Québec. 
Fédération étudiante universitaire du Québec (FEUQ). (2011). Le travail rémunéré et les études universitaires. Une nouvelle norme de la vie universitaire. Montréal, Québec.

Forbus, P., Newbold, J. J., \& Mehta, S. S. (2010). A study of non-traditional and traditional students in terms of their time management behaviors, stress factors, and coping strategies. Proceeding of the Academy of Educational Leadership, 15(2), 67-71.

Gauthier, M., \& Vultur, M. (2007). Les valeurs des jeunes et leur impact sur les stratégies d'insertion professionnelle. Dans J.-P. Dupuis (dir.), Sociologie de l'entreprise ( $2^{\mathrm{e}}$ éd., p. 273-292). Montréal, Québec : Gaëtan Morin éditeur.

Gouvernement du Québec (2007a). Pour que l'égalité de droit devienne une égalité de fait. Plan d'action 2007-2010. Québec, Québec.

Gouvernement du Québec (2007b). Pour que l'égalité de droit devienne une égalité de fait. Politique gouvernementale pour l'égalité entre les femmes et les hommes. Québec, Québec.

Gouvernement du Québec (2011). Pour que l'égalité de droit devienne une égalité de fait. Plan d'action 2011-2015. Québec, Québec.

Groupe de réflexion et d'action sur la reconnaissance des acquis et des compétences (GRAC). (2010). Reconnaissance des acquis et des compétences : élément stratégique pour l'apprentissage tout au long de la vie. Québec, Québec : Université du Québec.

Groupe de travail sur la réalité de l'adulte à l'Université Laval. (2007). La réalité de l'adulte à l'Université Laval : vers l'apprentissage tout au long de la vie. Québec, Québec : Université Laval.

Groupe de travail sur le financement des universités (1997). Rapport du groupe de travail sur le financement des universités. Repéré à : http://collections.banq.qc.ca/ ark:/52327/bs40908.

Hamel, J., Méthot, C., \& Doré, G. (2011). Étudier et être étudiant, quelles valeurs pour les jeunes d'aujourd'hui. Dans J. Hamel, C. Pugeault-Cicchelli, O. Galland \& V. Cicchelli (dir.), La jeunesse n'est plus ce qu'elle était (p. 119-130). Rennes, France : Presses universitaires de Rennes.

Kim, Karen A., Sax, L. J., Lee, J. J., \& Hagedorn, L. S. (2010). Redefining nontraditional students: Exploring the self-perceptions of community college students. Community College Journal of Research and Practice, 34(5), 402-422.

Laplante, B., Street, M. C., Moulin, S., Doray, P., \& Verdy, J. (2010). Parcours scolaires et travail salarié : une perspective longitudinale (Note 7). Montréal, Québec : CAPRES, CIRST.

Lévy, P. (1997). La cyberculture. Rapport au Conseil de l'Europe. Paris, France : Éditions Odile Jacob.

Mercure, D., \& Vultur, M. (2010). La signification du travail : nouveau modèle productif et éthos du travail au Québec. Québec, Québec : Presses de l'Université Laval.

Ministère de l'Éducation (MEQ). (2000a). Politique québécoise à l'égard des universités : pour mieux assurer notre avenir collectif. Québec, Québec. 
Ministère de l'Éducation (MEQ). (200ob). Politique québécoise de financement des universités. Québec, Québec.

Ministère de l'Éducation (MEQ). (2002a). Plan d'action en matière d'éducation des adultes et de formation continue : apprendre tout au long de la vie. Québec, Québec.

Ministère de l'Éducation (MEQ). (2002b). Politique gouvernementale d'éducation des adultes et de formation continue : apprendre tout au long de la vie. Québec, Québec.

Ministère de l'Éducation, du Loisir et du Sport (MELS). (2007). Programme étudestravail de l'Aide financière aux études : évaluation. Québec, Québec.

Ministère de l'Emploi et de la Solidarité sociale, et Ministère de l'Éducation, du Loisir et du Sport (MESS, et MELS). (2008). Le Pacte pour l'emploi : le Québec de toutes ses forces. Québec, Québec.

Ministère de l'Emploi et de la Solidarité sociale (MESS). (2012). Rapport statistique sur les individus, entreprises et organismes participant aux interventions des services publics d'emploi, année 2011-2012. Québec, Direction de la statistique et de l'information de gestion.

Ministère de l'Enseignement supérieur, de la Recherche, de la Science et de la Technologie (MESRST). (2013). Renforcer la recherche ainsi que la collaboration entre les établissements et leurs milieux. Sommet sur l'enseignement supérieur, Québec, Québec.

Mouvement québécois des adultes en formation, et Fédération des associations étudiantes universitaires québécoises en éducation permanente (MQAF, et FAEUQEP). (2009). Une réforme de l'aide financière s'impose pour répondre aux besoins des étudiants adultes. Propositions au ministère de l'Éducation, du Loisir et du Sport et au Comité consultatif sur l'accessibilité financière aux études, Montréal, Québec.

Mouvementquébécoisdesadultes enformation, etFédérationdesassociationsétudiantes universitaires québécoises en éducation permanente (MQAF, et FAEUQEP). (2011). Des modifications à l'aide financière insuffisantes pour compenser les inconvénients de la hausse des droits de scolarité. Point de vue soumis au Comité consultatif sur l'accessibilité financière aux études, Montréal, Québec.

Pilote, A., \& Garneau, S. (2011). La contribution de l'entretien biographique à l'étude de l'hétérogénéité de l'expérience étudiante et de son évolution dans le temps. Recherches Sociologiques et Anthropologiques, 42(2), 11-30.

Pralong, J. (1999). Sur la notion de " rapport aux études » : une construction théorique et son illustration dans l'université de masse. Psychologie \& Éducation, 37, 75-85.

Roy, J. (2008). Entre la classe et les mcjobs : portrait d'une génération de cégépiens. Québec, Québec : Presses de l'Université Laval.

Tremblay, H. P., \& Roy, P. (2013). Rapport d'étape du chantier sur la politique de financement des universités. Québec, Québec.

UNESCO. (2005). Vers les sociétés du savoir. France.

Van der Maren, J.-M. (1996). Méthodes de recherche pour l'éducation ( $2^{\mathrm{e}}$ éd.). Montréal, Québec : Presses de l'Université de Montréal. 


\section{Auteures-ressources}

Mélanie Julien

Conseil supérieur de l'éducation

melanie.julien@cse.gouv.qc.ca

Mélanie Julien est titulaire d'une maîtrise en sociologie de l'Université Laval. Elle œuvre depuis 2000 au Conseil supérieur de l'éducation (CSE), organisme dont la mission est de conseiller l'État québécois sur toute question relative à l'éducation. Après plusieurs années à la Direction des études et de la recherche, où elle était chargée des études quantitatives et qualitatives, Mme Julien coordonne depuis 2007 les travaux de la Commission de l'enseignement et de la recherche universitaires. Ses plus récentes recherches ont porté sur la formation à distance, les étudiants universitaires, l'assurance qualité à l'enseignement universitaire, les formations aux cycles supérieurs et l'internationalisation des universités, menant chaque fois à la publication d'avis du CSE ou de rapports de recherche.

Lynda Gosselin est titulaire d'une maitrise en administration et politique scolaires de l'Université Laval où elle a aussi mené des études doctorales. Elle œuvre au Conseil supérieur del'Éducation (CSE) depuis 2008. Elle a auparavant été conseillère àla recherche à la Fédération des comités de parents du Québec et a exercé, pendant de nombreuses années, des fonctions d'agente de recherche et de spécialiste des sciences de l'éducation à l'Université Laval et à la Télé-Université. En plus de ses collaborations à la production des récents avis du CSE sur l'enseignement universitaire, elle a participé au cours de sa carrière à différentes activités de recherche, d'enseignement, de communication et de conception de cours dans des domaines tels que les théories de l'organisation, la formation à distance, la démocratie scolaire et l'évaluation en éducation. 